Article

\title{
The Relationship between Selected CNR1, MC4R, LEP, FTO and VDR Gene Polymorphisms and Several Basic Toxicological Parameters Among Persons Occupationally Exposed to Arsenic, Cadmium and Lead
}

\author{
Tomasz Matys ${ }^{1}$, Anna Szymańska-Chabowska ${ }^{1}$, Katarzyna Bogunia-Kubik ${ }^{2}$, Beata Smyk ${ }^{1}$, \\ Małgorzata Kamińska ${ }^{2}$, Grzegorz Mazur ${ }^{1}$ (D), Rafał Poręba ${ }^{1}$ and Paweł Gać ${ }^{3, *(D)}$ \\ 1 Department of Internal Medicine, Occupational Diseases, Hypertension and Clinical Oncology, \\ Wroclaw Medical University, Borowska 213, PL 50-556 Wroclaw, Poland; t.matys@interia.pl (T.M.); \\ aszyman@mp.pl (A.S.-C.); beata.smyk@umed.wroc.pl (B.S.); grzegorz.mazur@umed.wroc.pl (G.M.); \\ rafal.poreba@umed.wroc.pl (R.P.) \\ 2 Laboratory of Clinical Immunogenetics and Pharmacogenetics, Hirszfeld Institute of Immunology and \\ Experimental Therapy, Polish Academy of Sciences, Weigla 12, PL 53-114 Wroclaw, Poland; \\ katarzyna.bogunia-kubik@hirszfeld.pl (K.B.-K.); malgorzata.kaminska@hirszfeld.pl (M.K.) \\ 3 Department of Hygiene, Wroclaw Medical University, Mikulicza-Radeckiego 7, PL 50-368 Wroclaw, Poland \\ * Correspondence: pawelgac@interia.pl; Tel.: +48-71-784-1502; Fax.: +48-71-784-1503
}

Received: 24 February 2020; Accepted: 6 April 2020; Published: 7 April 2020

\begin{abstract}
The purpose of this work was to assess the influence of selected CNR1, MC4R, LEP, FTO and VDR FOKI gene polymorphisms on blood and urine concentration markers of lead, cadmium and arsenic in a population directly exposed to these metals. Eighty-five people exposed to lead, arsenic and cadmium were qualified to take part in the study. Standard urine samples and $25 \mathrm{~mL}$ of venous blood from each worker were collected to assay basic laboratory and toxicological markers as well as selected single nucleotide polymorphisms (SNPs) within CNR1—cannabinoid receptor 1 gene ( $r s 806368, r s 806381, r s 1049353, r s 12720071)$, MC4R - melanocortin 4 receptor gene (rs17782313), LEP-leptin promoter gene (rs7799039), FTO—alpha-ketoglutarate-dependent dioxygenase gene (rs9939609) and VDR-vitamin D receptor (rs10735810) genes. It appeared that, except for the MC4R SNP, all the other polymorphisms were found to be associated with various laboratory parameters. Arsenic concentration in urine was associated with all four CNR1 and LEP SNPs, while cadmium concentration in blood was affected by the VDR polymorphism. Moreover, some significant relationships were also observed between CNR1 rs1049353 and FTO rs9939609 gene variants and markers of lead exposure. These results imply SNPs within genes coding for proteins involved in development of metabolic syndrome may be of prognostic value for persons directly exposed to lead, cadmium and arsenic.
\end{abstract}

Keywords: arsenic; cadmium; lead; single nucleotide polymorphism; zinc protoporphyrin

\section{Introduction}

Metabolic syndrome is an important global clinical problem and a challenge for modern medicine [1]. Metabolic syndrome was firstly described in specified detail in 1988 by Reaven. He suggested that it has four components: visceral obesity, hyperglycemia, arterial hypertension and dyslipidemia, described as hyper triglycerides, low HDL cholesterol fraction and high non-HDL fraction $[2,3]$. 
Research that aims to explain the causes of metabolic syndrome and its epidemic occurrence is based, on the one hand, on genetic and genotypic analyses and, on the other hand, observation of influence of the external environment $[4,5]$.

Metabolic syndrome is multi-genetic. Among the genes with the strongest relationship with metabolic syndrome, CNR1-cannabinoid receptor 1 gene, LEP-leptin promoter gene, FTO-alpha-ketoglutarate-dependent dioxygenase gene, MC4R-melanocortin 4 receptor gene and VDR-vitamin D receptor stand out.

CNR1 (cannabinoid receptor 1) is a G protein-coupled receptor activated by endogenous and exogenous cannabinoids. They are found mostly in the central nervous system, in the cerebellum, nucleus accumbens and several other brain regions responsible for hunger, satiety and the reward system [6]. Several specific single nucleotide polymorphisms of the CNR1 gene (located in 6q15) were found ( $r s 806381, r s 806368, r s 1049353, r s 12720071)$, as well as several possible combinations. The most popular, A to $G$ transition in $r s 1049353$, leads to higher body mass index and wider waist circumference [7]. Another studied polymorphism was leptin (LEP), a hormone made mostly by adipocyte cells. It inhibits hunger and stimulates the sympathetic nervous system. Despite its seemingly straightforward effect on the human organism, current research suggests that leptin polymorphism is not a relevant obesity marker [8]. Next, MC4R polymorphism was studied. Melanocortin 4 receptor is a known genetic obesity marker [9]. Yet, the mechanism remains unclear, and homozygous CC ( $r s 17782313$ ) tends to be associated with higher body mass index and insulin resistance [10]. Another typical obesity polymorphism is the FTO gene responsible for fat mass and an obesity-associated protein also known as alpha-ketoglutarate-dependent dioxygenase. Certain variants of this enzyme, active mostly in the central nervous system, are also correlated with higher BMI and obesity [11]. Finally, VDR (vitamin D receptor) FokI polymorphism was studied. This particular receptor is responsible for most of the comprehensive activity of vitamin D. Some variants are known to be responsible for different bone density, some are correlated with renin activity and some could even directly induce obesity [12].

According to current knowledge, exposure to metals may affect the development of metabolic syndrome. There are more and more reports confirming the existence of such a relationship.

Using data from the 2011-2014 National Health and Nutrition Examination Survey, Bulka et al. evaluated associations between essential and toxic metals exposure and metabolic syndrome [13]. The positive correlations observed for arsenic exposure were due to an elevated prevalence of high blood pressure, low HDL cholesterol and high triglycerides among people with greater exposures. On the other hand, greater lead and cadmium co-exposures were related to a lower prevalence of dyslipidemia and abdominal obesity.

On the contrary, an analysis based on the Korea National Health and Nutrition Examination Survey (KNHANES) found that a higher prevalence of metabolic syndrome was associated with higher blood lead levels in the Korean population [14].

In a study by Wang et al., blood and urinary markers of 18 heavy metals among 9537 adults in NHANES 2003-2014 were evaluated. This study suggests that cumulative exposure to heavy metals as mixtures is associated with obesity and its related to chronic conditions such as hypertension and diabetes type II [15].

Luzhetskyi and co-authors proved that children with higher serum levels of cadmium and arsenic (1.4-2.0 times vs. the reference group) demonstrated 2.2 times more frequent endocrine diseases, up to 2.7 times more frequent obesity-related diseases, when compared to the reference group. Metabolic disorders in that group were associated with some lipid metabolism changes [16].

Similarly, study of Kawakami et al. demonstrated that exposure to cadmium caused a reduction of adipocyte size and the modulation of adipokine expression. The reduction in adipocyte size by $\mathrm{Cd}$ may arise from an imbalance between lipid synthesis and lipolysis. In addition, the expression levels of leptin, adiponectin and resistin decreased in adipocytes. So, exposure to $\mathrm{Cd}$ may induce unusually small adipocytes and modulate the expression of adipokines differently from the case of 
physiologically small adipocytes, and it may accelerate the risk of developing insulin resistance and type 2 diabetes [17].

Numerous data concerning potential arsenic, cadmium and lead effects on development of obesity and metabolic syndrome have inspired us to pose a hypothesis that concentrations of these metals in people occupationally exposed are related to selected gene polymorphisms and determine metabolic disorders.

The purpose of this work was to assess the influence of selected single nucleotide polymorphisms (SNPs) coding for proteins involved in development of metabolic syndrome with the previously mentioned selected gene polymorphisms on blood and urine concentration markers of lead, cadmium and arsenic in a population directly exposed to these metals.

\section{Materials and Methods}

Eighty-five people, employees of a copper smelter and refinery, were qualified to be in the study. Inclusion criteria for the study were employment in workplaces exposed to arsenic, cadmium and lead (metal concentrations $>0.1$ maximum admissible concentration (MAC)) and work-related exposure to metals for at least 0.25 years. Size and quality of environmental exposure for all subjects included in the study were similar because all subjects resided in the same region. Only people who lived in the region for a long time (for at least 3 years) were included in the study group. The data and biological sample were collected in June 2016. There were 67 men and 18 women, and age ranged from 26 to 67 years. Thirty-three participants were obese, 34 of them were previously diagnosed with hypertension and 37 of them were smokers. Full clinical characteristics of the group are shown in Table 1, and basic laboratory characteristics of the population are shown in Table 2.

Table 1. Clinical characteristics of the study population.

\begin{tabular}{|c|c|c|c|c|c|}
\hline & $X$ & $\mathrm{Me}$ & SD & Min & $\operatorname{Max}$ \\
\hline Age (years) & 49.04 & 50.00 & 11.08 & 26.00 & 67.00 \\
\hline Height $(\mathrm{cm})$ & 174.24 & 175.50 & 7.64 & 156.00 & 190.00 \\
\hline Weight (kg) & 87.39 & 86.00 & 15.93 & 53.00 & 127.00 \\
\hline BMI $\left(\mathrm{kg} / \mathrm{m}^{2}\right)$ & 28.68 & 27.91 & 4.29 & 19.00 & 40.90 \\
\hline Waist circumference $(\mathrm{cm})$ & 100.44 & 100.00 & 12.55 & 72.00 & 125.00 \\
\hline \multirow[t]{2}{*}{ Pack-years } & 465.41 & 340.00 & 386.40 & 60.00 & 1500.00 \\
\hline & \multicolumn{2}{|c|}{$\mathrm{n}$} & \multicolumn{3}{|c|}{$\%$} \\
\hline Number & \multicolumn{2}{|c|}{85} & \multicolumn{3}{|c|}{100.0} \\
\hline \multicolumn{6}{|l|}{ Gender } \\
\hline Male & \multicolumn{2}{|c|}{67} & \multicolumn{3}{|c|}{78.8} \\
\hline Female & \multicolumn{2}{|c|}{18} & \multicolumn{3}{|c|}{21.2} \\
\hline \multicolumn{6}{|l|}{ Weight } \\
\hline Normal & \multicolumn{2}{|c|}{18} & \multicolumn{3}{|c|}{21.2} \\
\hline Overweight & \multicolumn{2}{|c|}{33} & \multicolumn{3}{|c|}{38.8} \\
\hline Obese & \multicolumn{2}{|c|}{33} & \multicolumn{3}{|c|}{38.8} \\
\hline Smokers & \multicolumn{2}{|c|}{37} & \multicolumn{3}{|c|}{43.5} \\
\hline Hypertension & \multicolumn{2}{|c|}{34} & \multicolumn{3}{|c|}{40.0} \\
\hline Diabetes & \multicolumn{2}{|c|}{10} & \multicolumn{3}{|c|}{11.8} \\
\hline
\end{tabular}

Max-maximal value; Me-median value; Min-minimal value; SD-standard deviation; X-arithmetic mean. 
Table 2. Conventional lab tests and calcium-phosphate balance in the study population.

\begin{tabular}{cccccc}
\hline & $\mathbf{X}$ & Me & SD & Min & Max \\
\hline WBC $(\mathrm{K} / \mu \mathrm{L})$ & 7.25 & 6.85 & 1.83 & 3.83 & 13.37 \\
\hline RBC $(\mathrm{M} / \mu \mathrm{L})$ & 5.04 & 5.05 & 0.36 & 4.28 & 5.85 \\
\hline Hemoglobin $(\mathrm{g} / \mathrm{dL})$ & 15.21 & 15.30 & 1.01 & 12.10 & 17.00 \\
\hline Hematocrit $(\%)$ & 44.58 & 44.70 & 2.70 & 37.90 & 50.00 \\
\hline Platelets $(\mathrm{K} / \mu \mathrm{L})$ & 249.93 & 246.00 & 52.44 & 129.00 & 406.00 \\
\hline Glucose $(\mathrm{mg} / \mathrm{dL})$ & 96.70 & 93.00 & 20.80 & 68.00 & 181.00 \\
\hline HbA1C $(\%)$ & 5.71 & 5.40 & 1.21 & 4.60 & 13.60 \\
\hline Total cholesterol $(\mathrm{mg} / \mathrm{dL})$ & 234.24 & 231.00 & 49.34 & 102.00 & 405.00 \\
\hline HDL cholesterol $(\mathrm{mg} / \mathrm{dL})$ & 51.02 & 49.00 & 11.08 & 27.00 & 86.00 \\
\hline LDL cholesterol $(\mathrm{mg} / \mathrm{dL})$ & 138.00 & 134.00 & 41.43 & 27.00 & 311.00 \\
\hline Triglycerides $(\mathrm{mg} / \mathrm{dL})$ & 236.58 & 196.00 & 155.31 & 46.00 & 824.00 \\
\hline Calcium $(\mathrm{mg} / \mathrm{dL})$ & 9.69 & 9.70 & 0.33 & 9.10 & 10.60 \\
\hline Phosphorus $(\mathrm{mg} / \mathrm{dL})$ & 3.43 & 3.30 & 0.66 & 2.20 & 5.60 \\
\hline 25-OH-D $(\mu \mathrm{d} / \mathrm{L})$ & 20.81 & 20.49 & 7.69 & 5.21 & 45.00 \\
\hline Parathormone $(\mathrm{ng} / \mathrm{L})$ & 46.68 & 43.30 & 20.02 & 15.50 & 108.60 \\
\hline
\end{tabular}

All the participants were asked to fill in a questionnaire about their medical history and lifestyle. Next, basic anthropometric measurements were taken. We also took standard urine samples and 25 $\mathrm{mL}$ of venous blood from each worker just after finishing their work shift to assay basic laboratory and toxicology markers as well as selected single nucleotide polymorphisms.

Blood count, fasting glucose, HbA1C, lipids (total cholesterol, HDL and LDL cholesterol, triglycerides) and calcium-phosphate balance markers (calcium, phosphorus, 25-OH- $\mathrm{D}_{3}$ and parathormone) were determined by standard methods in accordance with the manufacturer's instructions.

We also determined concentrations of lead (Pb-B), cadmium (Cd-B), zinc protoporphyrins in blood (ZnPP) and arsenic in urine (As-U). Blood lead and cadmium concentrations were measured by graphite furnace atomic absorption spectrometry Solaar M6 (Thermo Elemental, UK). The calibration curves of lead and cadmium were prepared with blood standards of certified reference material (BCR IRMM). Both methods are routinely monitored by determination of reference material (Recipe) and participation in an intercomparison program for toxicological analyses in biological materials, G-EQUAS. The measurement was calculated as micrograms per liter $(\mu \mathrm{g} / \mathrm{L})$, and the biological exposure limits were $500 \mu \mathrm{g} / \mathrm{L}$ for lead and $5 \mu \mathrm{g} / \mathrm{L}$ for cadmium according to recommendations of the National Hygiene Institute and Institute of Occupational Health. ZnPP was measured using a rapid fluorometric screening method by means of Hematofluorymeter ProtoFluor (Helena Laboratories, Beaumont, Texas, USA). Total urine arsenic concentration was measured by Hydride Generation Atomic Absorption Spectrometry (HGAAS) using the VP100 Continuous Flow Vapour System. To determine the calibration curve, the reference material $\mathrm{ClinCal}{ }^{\circledR}$ Urine Calibrator (Recipe) was used. We monitored the accuracy of the analytical method by analyzing samples of a reference material, Seronorm Trace Elements Urine (SERO AS, Oslo Norway), and participation in an intercomparison program for toxicological analyses in biological materials, G-EQUAS. The biological exposure limit proposed by the National Hygiene Institute and Institute of Occupational Health for arsenic in urine is $35 \mu \mathrm{g} / \mathrm{L}(35 \mu \mathrm{g} / \mathrm{g}$ creatinine).

In the study we analyzed selected single nucleotide polymorphisms (SNPs) for cannabinoid receptor 1 gene (CNR1), melanocortin 4 receptor gene (MC4R), leptin (LEP), alpha-ketoglutarate-dependent dioxygenase gene (FTO) and vitamin D receptor FokI. In the present study, the following eight SNPs were selected: $r s 17782313(\mathrm{~T}>\mathrm{C})$, located upstream of the MC4R gene 
on chromosome 18q22; rs7799039 (G>A), a common promoter polymorphic site within the LEP gene on chromosome 7q31.3; rs9939609 (G>A), a SNP within intron 1 of the FTO gene on chromosome 16q12.2; rs10735810 (FokI) (C>T), located within exon 2 of the VDR gene on chromosome 12q11; and four polymorphisms of the CNR1 gene located on chromosome 6q15 (one intronic SNP rs806381 A>G, $r s 806368 \mathrm{~T}>\mathrm{C}$ within $3^{\prime} \mathrm{UTR}, r s 1049353$ a synonymous $\mathrm{G}>\mathrm{A}$ polymorphism (within the splicing site), and $r s 12720071 \mathrm{~A}>\mathrm{G}$, a substitution in 3'UTR). Genotyping was performed by real-time polymerase chain reaction (PCR) amplifications and a melting curve analysis using a LightSNiP typing assay (TIB-MolBiol, Berlin, Germany). Real-time PCR was carried out on a LightCycler 480 Real-Time PCR system (Roche Diagnostics, Rotkreuz, Switzerland) in accordance with the conditions recommended by the manufacturers.

The research was compliant with Good Clinical Practice guidelines, The Declaration of Helsinki and was based on consent from a local Bioethical Committee (No KB-398/2018, date: 25.06.2018).

Statistical analyses were calculated using the statistical program STATISTICA 13 (Dell Inc., Tulsa, Oklahoma, USA). For the quantitative variables, the arithmetic mean (X), the median value (Me), the standard deviation (SD) as well as the minimal (Min) and maximal (Max) values of assayed parameters were calculated in the studied groups. Distribution of variables was tested using the Lilliefors and W-Shapiro-Wilk tests. In the case of independent, quantitative variables having normal distributions, a $t$ test for independent variables and the analysis of variances ANOVA (unifactorial parametric) were used in the further statistical analysis. The U test of Mann-Whitney or a non-parametric equivalent of Kruskal-Wallis ANOVA analysis of variance test were used in the case of variables with non-normal distributions. The significant differences between the arithmetic means were estimated using a post-hoc Newman-Keuls test. Results for the nominal variables were presented in percentages. In order to assess the relations between studied variables, a correlation analysis was performed. In the case of variables having a normal distribution, Pearson's $r$ was calculated, and for the variables with a distribution other than normal, the Spearman's $r$ correlation coefficient was used. Results at the level of $p<0.05$ were regarded as statistically significant.

\section{Results}

The mean blood calcium, phosphorus, 25-hydroxyvitamin $\mathrm{D}$ and parathormone concentrations in the occupationally exposed group were accordingly $9.69 \pm 0.33 \mathrm{mg} / \mathrm{dL}, 3.43 \pm 0.66 \mathrm{mg} / \mathrm{dL}, 20.81 \pm 7.69$ $\mu \mathrm{g} / \mathrm{L}$ and $46.68 \pm 20.02 \mathrm{ng} / \mathrm{L}$. These are presented in Table 2.

Arsenic concentration was $11.74 \pm 9.37 \mu \mathrm{g} / \mathrm{L}$, cadmium was $0.84 \pm 0.80 \mu \mathrm{g} / \mathrm{L}$, lead was 199.23 $\pm 117.02 \mu \mathrm{g} / \mathrm{L}$ and $\mathrm{ZnPP}$ was $47.94 \pm 30.64 \mu \mathrm{g} / \mathrm{dL}$. A total of $3.5 \%$ of employees had a urine arsenic concentration higher than the norm of the allowable concentration in biological material (determined as a maximum of $35 \mu \mathrm{g} / \mathrm{L}$ ). Totals of $1.2 \%$ and $16.5 \%$ of workers, respectively, had a blood lead concentration and blood zinc protoporphyrin concentration higher than the norms of the allowable concentration in biological material (determined as maximums of 500 and $70 \mu \mathrm{g} / \mathrm{L}$, respectively). Nobody in the study group was characterized by exceeding the norm of the permissible blood cadmium concentration. These are presented in Table 3.

Distributions of alleles and genotypes of selected SNPs in the studied population are shown in Table 4. Most genotypes had frequencies exceeding 10\%. The CRN1 rs12720071 GG homozygosity was the rarest and was detected in one individual only (1.2\%). The other rare genotypes were MC4R rs17782313 GG and CNR1 rs1049353 AA homozygosity observed both in five (5.9\%) cases, and the CNR1 rs806386 CC genotype was found in seven (8.2\%) cases. These distributions closely resemble those described for other European populations. 
Table 3. Basic toxicological parameters in the study population.

\begin{tabular}{|c|c|c|c|c|c|}
\hline & $X$ & Me & SD & Min & $\operatorname{Max}$ \\
\hline Exposure period (years) & 17.64 & 13.00 & 13.33 & 0.25 & 46.00 \\
\hline As-U $(\mu \mathrm{g} / \mathrm{L})$ & 11.74 & 9.93 & 9.37 & 0.27 & 46.15 \\
\hline Cd-B $(\mu \mathrm{g} / \mathrm{L})$ & 0.84 & 0.55 & 0.80 & 0.22 & 4.61 \\
\hline $\mathrm{Pb}-\mathrm{B}(\mu \mathrm{g} / \mathrm{L})$ & 199.23 & 193.80 & 117.02 & 22.20 & 520.90 \\
\hline $\mathrm{ZnPP}(\mu \mathrm{g} / \mathrm{dL})$ & 47.94 & 35.00 & 30.64 & 21.00 & 160.00 \\
\hline & \multicolumn{2}{|c|}{$n$} & \multicolumn{3}{|c|}{$\%$} \\
\hline Studied population & \multicolumn{2}{|c|}{85} & \multicolumn{3}{|c|}{100.0} \\
\hline $\begin{array}{l}\text { As-U >acceptable biological } \\
\text { concentration }(>35 \mu \mathrm{g} / \mathrm{L})\end{array}$ & \multicolumn{2}{|c|}{3} & \multicolumn{3}{|c|}{3.5} \\
\hline $\begin{array}{l}\text { Cd-B >acceptable biological } \\
\text { concentration }(>5 \mu \mathrm{g} / \mathrm{L})\end{array}$ & \multicolumn{2}{|c|}{0} & \multicolumn{3}{|c|}{0.0} \\
\hline $\begin{array}{l}\mathrm{Pb}-\mathrm{B}>\text { acceptable biological } \\
\text { concentration }(>500 \mu \mathrm{g} / \mathrm{L})\end{array}$ & \multicolumn{2}{|c|}{1} & \multicolumn{3}{|c|}{1.2} \\
\hline $\begin{array}{l}\mathrm{ZnPP}>\text { acceptable biological } \\
\text { concentration }(>70 \mu \mathrm{g} / \mathrm{dL})\end{array}$ & \multicolumn{2}{|c|}{14} & \multicolumn{3}{|c|}{16.5} \\
\hline
\end{tabular}

Table 4. Selected polymorphisms of genes CNR1, MC4R, LEP, FTO and VDR FokI in the study population.

\begin{tabular}{|c|c|c|c|c|c|c|}
\hline SNP & Genotype & $n$ & $\%$ & Allele & $n$ & $\%$ \\
\hline \multirow{3}{*}{$\begin{array}{c}\text { rs806381 } \\
\text { gene CNR1 }\end{array}$} & homozygote AA & 27 & 31.8 & \multirow{3}{*}{$\begin{array}{l}\text { allele A } \\
\text { allele G }\end{array}$} & \multirow{3}{*}{$\begin{array}{l}70 \\
58\end{array}$} & \multirow{3}{*}{$\begin{array}{l}82.4 \\
68.2\end{array}$} \\
\hline & heterozygote AG & 43 & 50.6 & & & \\
\hline & homozygote GG & 15 & 17.6 & & & \\
\hline \multirow{3}{*}{$\begin{array}{c}\text { rs806368 } \\
\text { gene CNR1 }\end{array}$} & homozygote CC & 7 & 8.2 & \multirow{3}{*}{$\begin{array}{l}\text { allele C } \\
\text { allele T }\end{array}$} & \multirow{3}{*}{$\begin{array}{l}30 \\
78\end{array}$} & \multirow{3}{*}{$\begin{array}{l}35.3 \\
91.8\end{array}$} \\
\hline & heterozygote CT & 23 & 27.1 & & & \\
\hline & homozygote TT & 55 & 64.7 & & & \\
\hline \multirow{3}{*}{$\begin{array}{c}\text { rs1049353 } \\
\text { gene CNR1 }\end{array}$} & homozygote AA & 5 & 5.9 & \multirow{3}{*}{$\begin{array}{l}\text { allele A } \\
\text { allele G }\end{array}$} & \multirow{3}{*}{$\begin{array}{l}39 \\
80\end{array}$} & \multirow{3}{*}{$\begin{array}{l}45.8 \\
94.1\end{array}$} \\
\hline & heterozygote AG & 34 & 40.0 & & & \\
\hline & homozygote GG & 46 & 54.1 & & & \\
\hline \multirow{3}{*}{$\begin{array}{l}\text { rs12720071 } \\
\text { gene CNR1 }\end{array}$} & homozygote AA & 69 & 81.2 & \multirow{3}{*}{$\begin{array}{l}\text { allele A } \\
\text { allele G }\end{array}$} & \multirow{3}{*}{$\begin{array}{l}84 \\
16\end{array}$} & \multirow{3}{*}{$\begin{array}{l}98.8 \\
18.8\end{array}$} \\
\hline & heterozygote AG & 15 & 17.6 & & & \\
\hline & homozygote GG & 1 & 1.2 & & & \\
\hline \multirow{3}{*}{$\begin{array}{c}\text { rs17782313 } \\
\text { gene MC4R }\end{array}$} & homozygote CC & 5 & 5.9 & \multirow{3}{*}{$\begin{array}{l}\text { allele C } \\
\text { allele T }\end{array}$} & \multirow{3}{*}{$\begin{array}{l}32 \\
80\end{array}$} & \multirow{3}{*}{$\begin{array}{l}37.6 \\
94.1\end{array}$} \\
\hline & heterozygote CT & 27 & 31.8 & & & \\
\hline & homozygote TT & 53 & 62.4 & & & \\
\hline \multirow{3}{*}{$\begin{array}{l}\text { rs7799039 } \\
\text { gene LEP }\end{array}$} & homozygote AA & 14 & 16.5 & \multirow{3}{*}{$\begin{array}{l}\text { allele A } \\
\text { allele G }\end{array}$} & \multirow{3}{*}{$\begin{array}{l}54 \\
70\end{array}$} & \multirow{3}{*}{$\begin{array}{l}63.5 \\
82.3\end{array}$} \\
\hline & heterozygote AG & 40 & 47.1 & & & \\
\hline & homozygote GG & 30 & 35.3 & & & \\
\hline \multirow{3}{*}{$\begin{array}{l}\text { rs9939609 } \\
\text { gene FTO }\end{array}$} & homozygote AA & 23 & 27.1 & \multirow{3}{*}{$\begin{array}{l}\text { allele A } \\
\text { allele T }\end{array}$} & \multirow{3}{*}{$\begin{array}{l}66 \\
62\end{array}$} & \multirow{3}{*}{$\begin{array}{l}77.6 \\
72.4\end{array}$} \\
\hline & heterozygote AT & 43 & 50.6 & & & \\
\hline & homozygote TT & 19 & 22.4 & & & \\
\hline \multirow{3}{*}{$\begin{array}{c}\text { rs10735810 } \\
\text { gene VDR FokI }\end{array}$} & homozygote CC & 25 & 29.4 & \multirow{3}{*}{$\begin{array}{l}\text { allele C } \\
\text { allele T }\end{array}$} & \multirow{3}{*}{$\begin{array}{l}66 \\
60\end{array}$} & \multirow{3}{*}{$\begin{array}{l}77.6 \\
70.6\end{array}$} \\
\hline & heterozygote CT & 41 & 48.2 & & & \\
\hline & homozygote TT & 19 & 22.4 & & & \\
\hline
\end{tabular}

The results of comparative analyses of arsenic urine concentrations of subgroups based on genotype criteria and alleles of single nucleotide polymorphisms of genes CNR1, MC4R, LEP, FTO and VDR FokI are presented in Table 5. We have proved that homozygosity AA in locus $r s 806381$ in the CNR1 gene is related to a statistically significant lower arsenic concentration, compared to 
heterozygosity AG and homozygosity GG, and the presence of allele G in this locus is associated with a significantly higher arsenic urine concentration. In locus rs12720071 of the CNR1 gene, homozygotes GG have statistically significant higher arsenic concentrations than heterozygotes AG and homozygotes $\mathrm{AA}$, and allele $\mathrm{A}$ in this locus can be associated with a statistically significant lower arsenic concentration. The analysis also has shown that allele A in locus $r s 1049353$ of the CNR1 gene can be responsible for lower arsenic concentrations, and allele G in locus rs7799039 of gene LEP is responsible for significantly higher arsenic concentrations in the studied population.

Table 5. Total arsenic concentration in subgroups divided according to selected polymorphisms of genes CNR1, MC4R, LEP, FTO and VDR FokI.

\begin{tabular}{|c|c|c|c|c|}
\hline SNP & Genotype & As-U ( $\mu \mathrm{g} / \mathrm{L})$ & Allele & As-U $(\mu \mathrm{g} / \mathrm{L})$ \\
\hline \multirow[t]{2}{*}{$\begin{array}{c}\text { rs806381 } \\
\text { gene CNR1 }\end{array}$} & $\begin{array}{l}\text { homozygote AA } \\
\text { heterozygote AG } \\
\text { homozygote GG }\end{array}$ & $\begin{array}{c}8.58 \pm 5.38 \\
12.81 \pm 10.00 \\
14.56 \pm 12.11\end{array}$ & $\begin{array}{l}\text { allele A } \\
\text { allele G }\end{array}$ & $\begin{array}{c}11.18 \pm 8.72 \\
13.24 \pm 10.47\end{array}$ \\
\hline & \multicolumn{2}{|c|}{$\begin{array}{l}\text { AA vs. AG: } p=0.044 \\
\text { AA vs. GG: } p=0.041\end{array}$} & \multicolumn{2}{|c|}{$\mathrm{G}(\mathrm{GG}$ or $\mathrm{AG})$ vs. non-G (AA): $p=0.032$} \\
\hline \multirow[t]{2}{*}{$\begin{array}{c}\text { rs } 806368 \\
\text { gene CNR1 }\end{array}$} & $\begin{array}{l}\text { homozygote CC } \\
\text { heterozygote } \mathrm{CT} \\
\text { homozygote } \mathrm{TT}\end{array}$ & $\begin{array}{c}11.43 \pm 9.61 \\
12.39 \pm 11.85 \\
11.51 \pm 8.31\end{array}$ & $\begin{array}{l}\text { allele C } \\
\text { allele T }\end{array}$ & $\begin{array}{c}12.19 \pm 11.27 \\
11.77 \pm 9.42\end{array}$ \\
\hline & \multicolumn{2}{|c|}{ ns } & \multicolumn{2}{|c|}{ ns } \\
\hline \multirow[t]{2}{*}{$\begin{array}{l}\text { rs1049353 } \\
\text { gene CNR1 }\end{array}$} & $\begin{array}{l}\text { homozygote AA } \\
\text { heterozygote AG } \\
\text { homozygote GG }\end{array}$ & $\begin{array}{c}8.99 \pm 4.68 \\
9.56 \pm 6.96 \\
13.70 \pm 10.91\end{array}$ & $\begin{array}{l}\text { allele A } \\
\text { allele } G\end{array}$ & $\begin{array}{c}9.48 \pm 6.66 \\
11.92 \pm 9.58\end{array}$ \\
\hline & \multicolumn{2}{|c|}{ ns } & \multicolumn{2}{|c|}{$\mathrm{A}(\mathrm{AA}$ or $\mathrm{AG})$ vs. non-A (GG): $p=0.039$} \\
\hline \multirow[t]{2}{*}{$\begin{array}{l}\text { rs } 12720071 \\
\text { gene CNR1 }\end{array}$} & $\begin{array}{l}\text { homozygote AA } \\
\text { heterozygote AG } \\
\text { homozygote GG }\end{array}$ & $\begin{array}{c}11.04 \pm 8.44 \\
13.72 \pm 12.26 \\
29.58 \pm 0.00\end{array}$ & $\begin{array}{l}\text { allele A } \\
\text { allele } G\end{array}$ & $\begin{array}{c}11.53 \pm 9.22 \\
14.72 \pm 12.49\end{array}$ \\
\hline & \multicolumn{2}{|c|}{$\begin{array}{l}\text { AA vs. GG: } p=0.034 \\
\text { AG vs. GG: } p=0.039\end{array}$} & \multicolumn{2}{|c|}{ A (AA or AG) vs. non-A (GG): $p=0.045$} \\
\hline \multirow[t]{2}{*}{$\begin{array}{l}\text { rs } 17782313 \\
\text { gene MC4R }\end{array}$} & $\begin{array}{l}\text { homozygote CC } \\
\text { heterozygote } \mathrm{CT} \\
\text { homozygote } \mathrm{TT}\end{array}$ & $\begin{array}{c}14.06 \pm 5.78 \\
10.25 \pm 8.36 \\
12.30 \pm 10.13\end{array}$ & $\begin{array}{l}\text { allele C } \\
\text { allele T }\end{array}$ & $\begin{array}{l}10.84 \pm 8.06 \\
11.60 \pm 9.56\end{array}$ \\
\hline & \multicolumn{2}{|c|}{ ns } & \multicolumn{2}{|c|}{ ns } \\
\hline \multirow[t]{2}{*}{$\begin{array}{l}\text { rs7799039 } \\
\text { gene LEP }\end{array}$} & $\begin{array}{l}\text { homozygote AA } \\
\text { heterozygote AG } \\
\text { homozygote GG }\end{array}$ & $\begin{array}{c}7.76 \pm 5.27 \\
12.11 \pm 9.98 \\
12.60 \pm 9.52\end{array}$ & $\begin{array}{l}\text { allele } A \\
\text { allele } G\end{array}$ & $\begin{array}{l}10.98 \pm 9.15 \\
12.31 \pm 9.72\end{array}$ \\
\hline & \multicolumn{2}{|c|}{ ns } & \multicolumn{2}{|c|}{$\mathrm{G}(\mathrm{GG}$ or $\mathrm{AG})$ vs. non-G (AA): $p=0.043$} \\
\hline \multirow[t]{2}{*}{$\begin{array}{l}\text { rs9939609 } \\
\text { gene FTO }\end{array}$} & $\begin{array}{l}\text { homozygote AA } \\
\text { heterozygote AT } \\
\text { homozygote TT }\end{array}$ & $\begin{array}{c}10.13 \pm 7.14 \\
13.10 \pm 10.87 \\
10.54 \pm 7.79\end{array}$ & $\begin{array}{l}\text { allele A } \\
\text { allele T }\end{array}$ & $\begin{array}{c}12.09 \pm 9.81 \\
12.31 \pm 10.04\end{array}$ \\
\hline & \multicolumn{2}{|c|}{ ns } & \multicolumn{2}{|c|}{ ns } \\
\hline \multirow[t]{2}{*}{$\begin{array}{l}\text { rs10735810 } \\
\text { gene VDR FokI }\end{array}$} & $\begin{array}{l}\text { homozygote CC } \\
\text { heterozygote CT } \\
\text { homozygote TT }\end{array}$ & $\begin{array}{c}13.58 \pm 11.47 \\
9.97 \pm 7.64 \\
13.21 \pm 9.54\end{array}$ & $\begin{array}{l}\text { allele C } \\
\text { allele T }\end{array}$ & $\begin{array}{l}11.34 \pm 9.36 \\
10.96 \pm 8.32\end{array}$ \\
\hline & \multicolumn{2}{|c|}{ ns } & \multicolumn{2}{|c|}{ ns } \\
\hline
\end{tabular}

ns-non-significant statistically.

The results of comparative analyses of cadmium blood concentrations of subgroups based on genotype criteria and alleles of single nucleotide polymorphisms of genes CNR1, MC4R, LEP, FTO and VDR FokI are presented in Table 6. We have proved that allele T in locus rs10735810 of VDR FokI gene can be a factor responsible for the significantly lower cadmium concentration in the studied population. 
Table 6. Cadmium concentration in subgroups divided according to selected polymorphisms of genes CNR1, MC4R, LEP, FTO and VDR FokI.

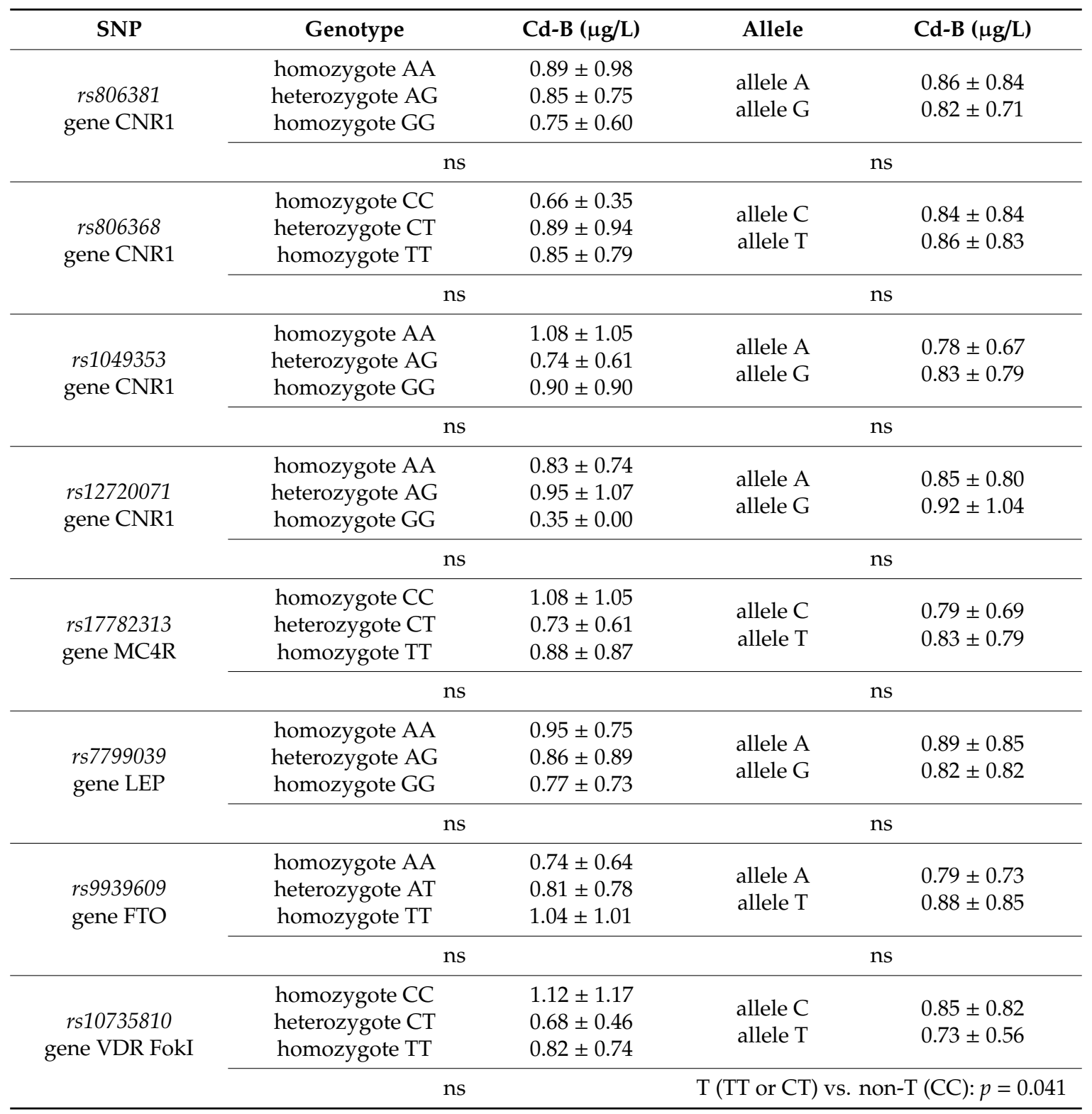

Similarly, the results of comparative analyses of lead blood concentrations of subgroups based on genotype criteria and alleles of single nucleotide polymorphisms of genes CNR1, MC4R, LEP, FTO and VDR FokI are presented in Table 7. In the studied population, homozygotes GG in locus rs 1049353 of the CNR1 gene have a significantly higher blood lead concentration compared to heterozygotes AG and homozygotes AA. The presence of allele A in the locus is correlated with a statistically relevant lower lead blood concentration. 
Table 7. Lead concentration in subgroups divided according to selected polymorphisms of genes CNR1, MC4R, LEP, FTO and VDR FokI.

\begin{tabular}{|c|c|c|c|c|}
\hline SNP & Genotype & $\mathrm{Pb}-\mathrm{B}(\mu \mathrm{g} / \mathrm{L})$ & Allele & $\mathrm{Pb}-\mathrm{B}(\mu \mathrm{g} / \mathrm{L})$ \\
\hline \multirow[t]{2}{*}{$\begin{array}{c}\text { rs806381 } \\
\text { gene CNR1 }\end{array}$} & $\begin{array}{l}\text { homozygote AA } \\
\text { heterozygote AG } \\
\text { homozygote GG }\end{array}$ & $\begin{array}{c}180.50 \pm 97.72 \\
202.85 \pm 137.05 \\
222.58 \pm 82.32\end{array}$ & $\begin{array}{l}\text { allele A } \\
\text { allele } \mathrm{G}\end{array}$ & $\begin{array}{l}194.23 \pm 123.09 \\
207.95 \pm 124.82\end{array}$ \\
\hline & \multicolumn{2}{|c|}{ ns } & \multicolumn{2}{|c|}{$\mathrm{ns}$} \\
\hline \multirow[t]{2}{*}{$\begin{array}{c}\text { rs } 806368 \\
\text { gene CNR1 }\end{array}$} & $\begin{array}{l}\text { homozygote CC } \\
\text { heterozygote CT } \\
\text { homozygote TT }\end{array}$ & $\begin{array}{c}207.09 \pm 50.81 \\
172.01 \pm 111.61 \\
209.61 \pm 124.52\end{array}$ & $\begin{array}{l}\text { allele C } \\
\text { allele T }\end{array}$ & $\begin{array}{l}180.20 \pm 101.05 \\
198.53 \pm 121.37\end{array}$ \\
\hline & \multicolumn{2}{|c|}{ ns } & \multicolumn{2}{|c|}{ ns } \\
\hline \multirow[t]{2}{*}{$\begin{array}{l}\text { rs1049353 } \\
\text { gene CNR1 }\end{array}$} & $\begin{array}{l}\text { homozygote AA } \\
\text { heterozygote AG } \\
\text { homozygote GG }\end{array}$ & $\begin{array}{l}171.58 \pm 117.21 \\
160.00 \pm 107.64 \\
231.23 \pm 116.39\end{array}$ & $\begin{array}{l}\text { allele A } \\
\text { allele G }\end{array}$ & $\begin{array}{l}161.48 \pm 107.35 \\
200.96 \pm 117.53\end{array}$ \\
\hline & \multicolumn{2}{|c|}{$\begin{array}{l}\text { AG vs. GG: } p=0.006 \\
\text { AA vs. GG: } p=0.045\end{array}$} & \multicolumn{2}{|c|}{$\begin{array}{c}\text { A (AA or } A G) \text { vs. non-A }(G G): p= \\
0.005\end{array}$} \\
\hline \multirow[t]{2}{*}{$\begin{array}{l}\text { rs12720071 } \\
\text { gene CNR1 }\end{array}$} & $\begin{array}{l}\text { homozygote AA } \\
\text { heterozygote AG } \\
\text { homozygote GG }\end{array}$ & $\begin{array}{c}193.85 \pm 115.37 \\
224.46 \pm 129.17 \\
192.20 \pm 0.00\end{array}$ & $\begin{array}{l}\text { allele A } \\
\text { allele } \mathrm{G}\end{array}$ & $\begin{array}{l}199.31 \pm 117.72 \\
222.44 \pm 125.05\end{array}$ \\
\hline & \multicolumn{2}{|c|}{ ns } & \multicolumn{2}{|c|}{ ns } \\
\hline \multirow[t]{2}{*}{$\begin{array}{l}\text { rs17782313 } \\
\text { gene MC4R }\end{array}$} & $\begin{array}{l}\text { homozygote CC } \\
\text { heterozygote CT } \\
\text { homozygote TT }\end{array}$ & $\begin{array}{c}244.16 \pm 99.42 \\
174.66 \pm 116.00 \\
207.51 \pm 118.55\end{array}$ & $\begin{array}{l}\text { allele C } \\
\text { allele T }\end{array}$ & $\begin{array}{l}185.52 \pm 114.97 \\
196.42 \pm 118.00\end{array}$ \\
\hline & \multicolumn{2}{|c|}{ ns } & \multicolumn{2}{|c|}{ ns } \\
\hline \multirow[t]{2}{*}{$\begin{array}{l}\text { rs7799039 } \\
\text { gene LEP }\end{array}$} & $\begin{array}{l}\text { homozygote AA } \\
\text { heterozygote AG } \\
\text { homozygote GG }\end{array}$ & $\begin{array}{l}208.89 \pm 137.93 \\
193.02 \pm 119.09 \\
201.17 \pm 108.88\end{array}$ & $\begin{array}{l}\text { allele A } \\
\text { allele } \mathrm{G}\end{array}$ & $\begin{array}{l}197.13 \pm 123.09 \\
196.51 \pm 114.08\end{array}$ \\
\hline & \multicolumn{2}{|c|}{$\mathrm{ns}$} & \multicolumn{2}{|c|}{ ns } \\
\hline \multirow[t]{2}{*}{$\begin{array}{l}\text { rs9939609 } \\
\text { gene FTO }\end{array}$} & $\begin{array}{l}\text { homozygote AA } \\
\text { heterozygote AT } \\
\text { homozygote TT }\end{array}$ & $\begin{array}{l}205.35 \pm 107.83 \\
206.36 \pm 127.10 \\
175.69 \pm 106.02\end{array}$ & $\begin{array}{l}\text { allele A } \\
\text { allele T }\end{array}$ & $\begin{array}{l}206.01 \pm 119.89 \\
196.96 \pm 121.01\end{array}$ \\
\hline & \multicolumn{2}{|c|}{ ns } & \multicolumn{2}{|c|}{ ns } \\
\hline \multirow[t]{2}{*}{$\begin{array}{l}\text { rs10735810 } \\
\text { gene VDR FokI }\end{array}$} & $\begin{array}{l}\text { homozygote CC } \\
\text { heterozygote } \mathrm{CT} \\
\text { homozygote } \mathrm{TT}\end{array}$ & $\begin{array}{c}224.20 \pm 139.97 \\
175.76 \pm 107.16 \\
217.04 \pm 99.02\end{array}$ & $\begin{array}{l}\text { allele C } \\
\text { allele T }\end{array}$ & $\begin{array}{l}194.11 \pm 121.91 \\
188.83 \pm 105.60\end{array}$ \\
\hline & \multicolumn{2}{|c|}{ ns } & \multicolumn{2}{|c|}{ ns } \\
\hline
\end{tabular}

The results of comparative analyses of zinc protoporphyrin blood concentrations of subgroups based on genotype criteria and alleles of single nucleotide polymorphisms of genes CNR1, MC4R, LEP, FTO and VDR FokI are presented in Table 8. It was proven that heterozygosity AG in locus rs1049353 of the CNR1 gene may result in a statistically lower ZnPP concentration compared to homozygosity AA and GG, and allele A is responsible for lower ZnPP concentrations. Apart from that, we documented that allele A in locus rs9939609 of the FTO gene is responsible for higher ZnPP concentration. 
Table 8. Zinc protoporphyrins (ZnPP) concentration in subgroups divided according to selected polymorphisms of genes CNR1, MC4R, LEP, FTO and VDR FokI.

\begin{tabular}{|c|c|c|c|c|}
\hline SNP & Genotype & $\mathrm{ZnPP}(\mu \mathrm{g} / \mathrm{dL})$ & Allele & $\mathrm{ZnPP}(\mu \mathrm{g} / \mathrm{dL})$ \\
\hline \multirow{4}{*}{$\begin{array}{c}\text { rs806381 } \\
\text { gene CNR1 }\end{array}$} & homozygote AA & $42.48 \pm 25.15$ & \multirow{3}{*}{$\begin{array}{l}\text { allele } A \\
\text { allele } G\end{array}$} & \multirow{3}{*}{$\begin{array}{l}45.99 \pm 27.84 \\
50.48 \pm 32.78\end{array}$} \\
\hline & heterozygote AG & $48.19 \pm 29.47$ & & \\
\hline & homozygote GG & $57.07 \pm 41.30$ & & \\
\hline & \multicolumn{2}{|c|}{ ns } & \multicolumn{2}{|c|}{ ns } \\
\hline \multirow{4}{*}{$\begin{array}{c}\text { rs806368 } \\
\text { gene CNR1 }\end{array}$} & homozygote CC & $64.86 \pm 48.64$ & \multirow{3}{*}{$\begin{array}{l}\text { allele C } \\
\text { allele T }\end{array}$} & \multirow{3}{*}{$\begin{array}{l}47.10 \pm 32.03 \\
46.42 \pm 28.49\end{array}$} \\
\hline & heterozygote CT & $41.70 \pm 24.01$ & & \\
\hline & homozygote TT & $48.40 \pm 30.15$ & & \\
\hline & \multicolumn{2}{|c|}{ ns } & \multicolumn{2}{|c|}{ ns } \\
\hline \multirow{5}{*}{$\begin{array}{l}\text { rs1049353 } \\
\text { gene CNR1 }\end{array}$} & homozygote AA & $56.60 \pm 42.48$ & \multirow{3}{*}{$\begin{array}{l}\text { allele A } \\
\text { allele G }\end{array}$} & \multirow{3}{*}{$\begin{array}{l}39.64 \pm 23.11 \\
47.40 \pm 30.03\end{array}$} \\
\hline & heterozygote AG & $37.15 \pm 18.61$ & & \\
\hline & homozygote GG & $54.98 \pm 34.51$ & & \\
\hline & \multirow{2}{*}{\multicolumn{2}{|c|}{$\begin{array}{l}\text { AG vs. GG: } p=0.009 \\
\text { AA vs. AG: } p=0.037\end{array}$}} & \multirow{2}{*}{\multicolumn{2}{|c|}{ A (AA or AG) vs. non-A (GG): $p=0.020$}} \\
\hline & & & & \\
\hline \multirow{4}{*}{$\begin{array}{l}\text { rs12720071 } \\
\text { gene CNR1 }\end{array}$} & homozygote AA & $47.38 \pm 30.92$ & \multirow{3}{*}{$\begin{array}{l}\text { allele A } \\
\text { allele G }\end{array}$} & \multirow{3}{*}{$\begin{array}{l}47.39 \pm 30.40 \\
50.38 \pm 30.26\end{array}$} \\
\hline & heterozygote AG & $47.47 \pm 28.91$ & & \\
\hline & homozygote GG & $94.00 \pm 0.00$ & & \\
\hline & \multicolumn{2}{|c|}{ ns } & \multicolumn{2}{|c|}{ ns } \\
\hline \multirow{4}{*}{$\begin{array}{l}\text { rs17782313 } \\
\text { gene MC4R }\end{array}$} & homozygote CC & $51.20 \pm 43.25$ & \multirow{3}{*}{$\begin{array}{l}\text { allele C } \\
\text { allele T }\end{array}$} & \multirow{3}{*}{$\begin{array}{l}45.88 \pm 32.92 \\
47.74 \pm 30.05\end{array}$} \\
\hline & heterozygote CT & $44.89 \pm 31.59$ & & \\
\hline & homozygote TT & $49.19 \pm 29.43$ & & \\
\hline & \multicolumn{2}{|c|}{ ns } & \multicolumn{2}{|c|}{ ns } \\
\hline \multirow{4}{*}{$\begin{array}{l}\text { rs7799039 } \\
\text { gene LEP }\end{array}$} & homozygote AA & $53.57 \pm 32.82$ & \multirow{3}{*}{$\begin{array}{l}\text { allele A } \\
\text { allele G }\end{array}$} & \multirow{3}{*}{$\begin{array}{l}46.53 \pm 30.42 \\
45.56 \pm 27.82\end{array}$} \\
\hline & heterozygote AG & $42.75 \pm 25.72$ & & \\
\hline & homozygote GG & $51.57 \pm 35.59$ & & \\
\hline & \multicolumn{2}{|c|}{ ns } & \multicolumn{2}{|c|}{ ns } \\
\hline & homozygote AA & $52.26 \pm 37.44$ & & \\
\hline rs9939609 & heterozygote AT & $50.44 \pm 29.70$ & allele A & $\begin{array}{l}51.08 \pm 32.33 \\
4634+2789\end{array}$ \\
\hline gene FTO & homozygote TT & $37.05 \pm 21.13$ & & \\
\hline & & & $\mathrm{A}(\mathrm{AA}$ or $\mathrm{AT}$ & $\mathrm{l}-\mathrm{A}(\mathrm{TT}): p=0.038$ \\
\hline & homozygote CC & $52.96 \pm 29.89$ & & \\
\hline rs10735810 & heterozygote CT & $42.17 \pm 28.05$ & $\begin{array}{l}\text { allele C } \\
\text { allele T }\end{array}$ & $45.85 \pm 30.95$ \\
\hline gene VDR FokI & homozygote TT & $53.79 \pm 35.97$ & & $40.00 \pm 30.90$ \\
\hline & & & & \\
\hline
\end{tabular}

In the correlation analysis we found statistically significant, linear correlations between cadmium concentration and white blood cell count $(\mathrm{r}=0.22, p=0.040), \mathrm{ZnPP}$ concentration and platelets $(\mathrm{r}=0.25$, $p=0.020)$, ZnPP concentration and phosphorus in blood $(\mathrm{r}=0.23, p=0.035)$ as well as ZnPP and vitamin $\mathrm{D}(\mathrm{r}=-0.22, p=0.032)$.

In the last part of the study, multivariate regression analysis was performed, and the following significant models were observed:

As-U $=1.330$ allele $\mathrm{G}$ in $r s 806381$ gene CNR1 - 4.274 allele A in $r s 1049353$ gene CNR1 - 18.415 allele A in rs12720071 gene CNR1 + 2.291 allele G in rs7799039 gene LEP + 0.424 BMI + 0.160 age + 5.324 diabetes \pm 4.877 .

$\mathrm{Cd}-\mathrm{B}=-0.439$ allele $\mathrm{T}$ in $r s 10735810$ gene VDR FokI +0.814 smoking -0.023 HDL cholesterol + $0.014 \mathrm{BMI} \pm 0.709$.

$\mathrm{Pb}-\mathrm{B}=-77.411$ allele $\mathrm{A}$ in $r s 1049353$ gene $\mathrm{CNR} 1+62.804$ hypertension +82.478 diabetes -44.553 phosphorus \pm 0.709 . 
Based on the obtained regression models it was shown that allele $\mathrm{G}$ in $r$ s806381 gene CNR1, allele $\mathrm{G}$ in $r s 7799039$ gene LEP, higher BMI, older age and diabetes were independently associated with higher As-U concentration; while allele A in rs1049353 gene CNR1 and allele A in rs12720071 gene CNR1 were independently associated with lower As-U concentration. It was shown that allele $\mathrm{T}$ in $r s 10735810$ gene VDR FokI and higher HDL cholesterol concentration were independently associated with lower Cd-B concentration, while smoking and higher BMI were independently associated with higher Cd-B concentration. Finally, allele A in $r s 1049353$ gene CNR1 and higher phosphorus concentration were independently associated with a lower $\mathrm{Pb}-\mathrm{B}$ concentration, while hypertension and diabetes were independently associated with a higher $\mathrm{Pb}-\mathrm{B}$ concentration (Table 9).

Table 9. Results of estimation for the final model obtained from multivariate regression analysis.

\begin{tabular}{|c|c|c|c|c|c|c|}
\hline & Independent Variable & $\begin{array}{l}\text { Regression } \\
\text { Coefficient }\end{array}$ & $\begin{array}{c}\text { Standard error of } \\
\text { Regression } \\
\text { Coefficient }\end{array}$ & $p$-Value & $\begin{array}{l}p \text { Value of } \\
\text { the Model }\end{array}$ & $\begin{array}{l}\text { Standard Error } \\
\text { of the Model }\end{array}$ \\
\hline \multirow{7}{*}{$\begin{array}{c}\text { model for } \\
\text { As-U }(\mu \mathrm{g} / \mathrm{L})\end{array}$} & $\begin{array}{c}\text { allele G in rs806381 gene } \\
\text { CNR1 }\end{array}$ & 1.330 & 0.666 & 0.041 & \multirow{7}{*}{0.043} & \multirow{7}{*}{4.877} \\
\hline & $\begin{array}{l}\text { allele A in rs1049353 gene } \\
\text { CNR1 }\end{array}$ & -4.274 & 2.095 & 0.037 & & \\
\hline & $\begin{array}{c}\text { allele A in rs12720071 } \\
\text { gene CNR1 }\end{array}$ & -18.415 & 9.136 & 0.037 & & \\
\hline & $\begin{array}{c}\text { allele G in } r s 7799039 \text { gene } \\
\text { LEP }\end{array}$ & 2.291 & 1.072 & 0.045 & & \\
\hline & $\mathrm{BMI}\left(\mathrm{kg} / \mathrm{m}^{2}\right)$ & 0.424 & 0.173 & 0.025 & & \\
\hline & age (years) & 0.160 & 0.070 & 0.015 & & \\
\hline & diabetes & 5.324 & 2.269 & 0.026 & & \\
\hline \multirow{4}{*}{$\begin{array}{l}\text { model for } \\
\text { Cd-B }(\mu g / L)\end{array}$} & $\begin{array}{l}\text { allele } \mathrm{T} \text { in } r s 10735810 \\
\text { gene VDR FokI }\end{array}$ & -0.439 & 0.187 & 0.022 & \multirow{4}{*}{0.006} & \multirow{4}{*}{0.709} \\
\hline & smoking & 0.814 & 0.184 & 0.001 & & \\
\hline & HDL cholesterol (mg/dL) & -0.023 & 0.011 & 0.045 & & \\
\hline & BMI (kg/m2) & 0.014 & 0.002 & 0.046 & & \\
\hline \multirow{4}{*}{$\begin{array}{l}\text { model for } \\
\mathrm{Pb}-\mathrm{B}(\mu \mathrm{g} / \mathrm{L})\end{array}$} & $\begin{array}{l}\text { allele A in rs1049353 gene } \\
\text { CNR1 }\end{array}$ & -77.411 & 26.320 & 0.004 & \multirow{4}{*}{0.014} & \multirow{4}{*}{112.131} \\
\hline & hypertension & 62.804 & 39.182 & 0.014 & & \\
\hline & diabetes & 82.478 & 58.742 & 0.016 & & \\
\hline & phosphorus (mg/dL) & -44.553 & 21.391 & 0.041 & & \\
\hline
\end{tabular}

\section{Discussion}

The relationship between some metals and body mass, obesity development and central hunger regulation is widely discussed and confirmed in numerous studies. Many studies focus on genetic and epigenetic mechanisms of obesity evolution. In the study of Tyrrell et al., Pb-exposed animals showed elevated hepatic triglyceride levels and increased expression of the gluconeogenic genes PEPCK and glucose-6-phosphatase [18]. In cultured rat hepatoma cells, treatment with Pb stimulated PEPCK and glucose-6-phosphatase gene expression, suggesting a possible direct effect of $\mathrm{Pb}$ on hepatic gluconeogenic gene expression. Vidal et al. proved that elevated maternal blood Cd levels were associated with lower birth weight, and higher maternal blood Cd levels were also associated with lower methylation at the PEG3 or at the MEG3 in methylated regions of newborn DNA [19].

Our study is either the first or one of the very few studies trying to determine the relationship between single nucleotide polymorphisms of genes involved in development of metabolic syndrome and toxicological parameters. Although the literature is poor, we decided to proceed with selected genes as they are the best studied in the aspect of metabolic syndrome. We found several statistically important correlations. However, we were unable to compare our results with other studies, as there have been none. On the other hand, there are numerous studies concerning studied polymorphisms and various other parameters. A comprehensive discussion of the subject will extend the framework 
of this research. Therefore, we decided to discuss a few examples to show the scientific and clinical importance of our study, as well as the need to continue the research.

Until now, we were unable to find other studies showing correlations between CNR1 polymorphism locus rs1049353 and arsenic concentration. Moreover, data concerning this polymorphism and its correlation with BMI parameters are inconclusive and often contradictory [7]. In our research we were able to determine a significant correlation: allele $\mathrm{G}$ is correlated with higher arsenic concentration. This poses the following question: what is the reason for such correlation? Do people with allele G consume more food, as some studies show [20], therefore absorbing more arsenic and resulting in a higher arsenic concentration? Or, on the other hand, does a greater amount of fat tissue enable a higher arsenic concentration? Whatever the mechanism could be, we have found a genetic predisposition to higher arsenic concentration. This requires further study, especially in light of the growing obesity epidemic.

In our study we discovered that allele G in locus rs7799039 of gene LEP is correlated with a markedly higher arsenic concentration. The authors were not able to find any other research to support or undermine our result. Nevertheless, the leptin polymorphism has been heavily studied recently. Carriers of A allele in the studied locus tend to have lower LDL and total cholesterol [21]. Another recent study shows association between leptin polymorphism and coronary artery disease and hypertension [22]. This finally leads us to the question about the role of arsenic in development of metabolic syndrome and cardiovascular diseases. This role is yet to be determined.

We were also able to find that allele $\mathrm{T}$ in locus $r$ s10735810 of VDR FokI gene is associated with lower cadmium concentration. Again, it seems that it is the first attempt to assess this correlation. There are single studies on metals and VDR FokI polymorphism. In the study by Szymanska-Chabowska et al., they were able to determine an association between another locus of VDR FokI gene ( $r s 2228570$ ) and concentration of lead and ZnPP [23]. Our study provides more input on the matter, especially that some research suggests there is no simple connection between cadmium concentration and vitamin $\mathrm{D}$ concentration [24].

In studied population, we were able to determine a significant correlation between the CNR1 polymorphism and lead and $\mathrm{ZnPP}$ concentrations. Again, this is the first research proving this relation. However, polymorphism of CNR1 is being thoroughly studied. It was found that $r s 1049353$ polymorphism was associated with specific changes in brain morphology as well as with evolution of positive symptoms in schizophrenia [25]. This poses a question about the role of lead in the development of psychiatric disorders and brain reconstruction.

As for the linear correlation between cadmium concentration and white blood cell count, our research is compliant with many previous studies, including the most recent ones [26,27]. Correlations of $\mathrm{ZnPP}$ concentration and platelets, $\mathrm{ZnPP}$ concentration and phosphorus in blood as well as $\mathrm{ZnPP}$ and vitamin $\mathrm{D}$ were not found in previous studies [23].

Finally, it should be mentioned that, when analyzing multiple polymorphisms affecting a single variable (e.g., blood concentration of a particular metal), it is necessary to consider the possible compensatory effects of polymorphisms. In the current study, this compensation in individuals may be due to the effect of polymorphisms on urinary arsenic levels. If a person is a carrier of allele $G$ in loci $r s 806381$ CNR1 gene and rs7799039 LEP gene, and at the same time a carrier of allele A in loci rs1049353 and rs12720071 CNR1 gene, the increase in As-U concentration due to the impact of the first two alleles may be reduced as a result of the impact of the second two alleles.

The authors of this work see two main limitations of this study. First, there is little or no research to compare and confront our data. Our study was conducted with the highest standards; we found statistically significant correlations, but still, further research needs to be conducted to confirm our results. Secondly, our study is based on a relatively small population ( 85 persons) for genetic study. This results in poor representation of certain alleles (allele G in locus $r s 12720071$ CNR1-18.8\%; allele C in locus $r s 17782313 \mathrm{MC} 4 \mathrm{R}-37.6 \%$ ). As for the first allele, we were able to obtain statistically significant correlation. If statistically important correlations are found in a relatively small group, the correlation is strong and it will be even more visible in larger groups. Finally, showing specific correlations between 
polymorphisms and metal concentrations is just the first step in understanding their complex role in the human organism; yet, it is an important step forward.

Relationships that we found between some genetic polymorphisms and arsenic, lead and cadmium exposure levels may indicate their role in the promotion of obesity and metabolic disorders. These metals may be one of many environmental factors that, in an unfavorable genetic constellation, contribute to higher cardiovascular risk resulting from obesity, diabetes and atherogenic lipid profile. Immunological processes modulated by vitamin D also have their impact on this risk. The relationship between lower cadmium levels and polymorphism of one of the vitamin D receptors proves that this polymorphism is beneficial in reducing cardiovascular risk in persons occupationally exposed to cadmium.

\section{Conclusions}

(1) Single nucleotide polymorphisms within genes coding for proteins involved in development of metabolic syndrome may be of prognostic value for persons directly exposed to lead, cadmium and arsenic.

(2) In the group occupationally exposed to arsenic, cadmium and lead, certain associations between polymorphisms $r s 806381, r s 1049353$ and $r s 12720071$ of gene CNR1 and polymorphism rs7799039 of gen LEP and arsenic concentration in urine were acquired:

- Allele G in locus rs806381 CNR1 and locus rs7799039 LEP can be responsible for higher arsenic concentrations;

- Allele A in locus rs1049353 and rs12720071 CNR1 can be responsible for lower arsenic concentrations.

(3) Cadmium concentration in blood in people occupationally exposed can be determined by polymorphism of rs10735810 VDR FokI gene:

- Allele $\mathrm{T}$ in locus rs10735810 VDR FokI gene can be responsible for lower cadmium concentration.

(4) In people occupationally exposed to arsenic, cadmium and lead, there are certain interactions between polymorphisms rs1049353 gene CNR1 and rs9939609 gene FTO and markers of lead exposure (lead and zinc protoporphyrin in blood):

- Allele A in locus rs1049353 CNR1 gene can be responsible for lower lead and $\mathrm{ZnPP}$ concentrations;

- Allele A in locus rs9939609 FTO gene can be responsible for higher ZnPP concentration.

(5) Polymorphism rs17782813 MC4R gene, as the only one in our study, did not affect concentrations of selected markers amongst workers occupationally exposed to lead, cadmium and arsenic.

Author Contributions: Conceptualization, A.S.-C., R.P. and P.G; Data curation, A.S.-C. and P.G.; Funding acquisition, A.S.-C.; Investigation, T.M., K.B.-K., B.S. and M.K.; Methodology, A.S.-C.; Supervision, G.M. and R.P.; Writing-original draft, T.M.; Writing-review \& editing, A.S.-C., K.B.-K., R.P. and P.G. All authors have read and agree to the published version of the manuscript.

Funding: All funds for the study came from Wroclaw Medical University local grants.

Conflicts of Interest: The authors report no conflicts of interest.

\section{References}

1. Saklayen, M.G. The Global Epidemic of the Metabolic Syndrome. Curr. Hypertens. Rep. 2018, $20,12$. [CrossRef]

2. Taton, J.; Bernas, M. Metabolic syndrome-Controversies between academic debate and real world medical practice. Endokrynol. Otyłość Zaburzenia Przemiany Mater. 2008, 5, 13-26. 
3. Engin, A. The Definition and Prevalence of Obesity and Metabolic Syndrome. Adv. Exp. Med. Biol. 2017, 960, 1-17. [PubMed]

4. Jeong, H.S. The Relationship between Workplace Environment and Metabolic Syndrome. Int. J. Occup. Environ. Med. 2018, 9, 176-183. [CrossRef] [PubMed]

5. Ziki, M.D.A.; Mani, A. Metabolic syndrome: Genetic insights into disease pathogenesis. Curr. Opin. Lipidol. 2016, 27, 162-171. [CrossRef]

6. Hryhorowicz, S.; Walczak, M.; Zakerska-Banaszak, O.; Słomski, R.; Skrzypczak-Zielińska, M. Pharmacogenetics of cannabinoids. Eur. J. Drug Metabol. Pharmacokinet. 2017, 43, 1-12. [CrossRef]

7. Doris, J.M.; Millar, S.A.; Idris, I.; O'Sullivan, S.E. Genetic polymorphisms of the endocannabinoid system in obesity and diabetes. Diabetes Obes. Metabol. 2019, 21, 382-387. [CrossRef]

8. Yan, J.; Wang, X.; Tao, H.; Yang, W.; Luo, M.; Lin, F. Lack of association between leptin G-2548A polymorphisms and obesity risk: Evidence based on a meta-analysis. Obes. Res. Clin. Pract. 2015, 9, 389-397. [CrossRef]

9. Krashes, M.J.; Lowell, B.B.; Garfield, A.S. Melanocortin-4 receptor-regulated energy homeostasis. Nat. Neurosci. 2016, 19, 206-219. [CrossRef]

10. Tschritter, O.; Haupt, A.; Preiss, H. An obesity risk SNP (rs17782313) near the MC4R gene is associated with cerebrocortical insulin resistance in humans. J. Obes. 2011, 2011, 283153. [CrossRef]

11. Speakman, J.R. The fat mass and obesity related (FTO) gene: mechanisms of impact on obesity and energy balance. Curr. Obes. Rep. 2015, 4, 73-91. [CrossRef] [PubMed]

12. Wysoczańska-Klaczyńska, A.; Ślęzak, A.; Hetman, M.; Barg, E. The impact of VDR gene polymorphisms on obesity, metabolic changes, bone mass disorders and neoplastic processes. Pediatr. Endocrinol. Diabetes Metabol. 2018, 24, 96-105. [CrossRef] [PubMed]

13. Bulka, C.M.; Persky, V.W.; Daviglus, M.L.; Durazo-Arvizu, R.A.; Argos, M. Multiple metal exposures and metabolic syndrome: A cross-sectional analysis of the National Health and Nutrition Examination Survey 2011-2014. Environ. Res. 2019, 168, 397-405. [CrossRef] [PubMed]

14. Rhee, S.Y.; Hwang, Y.C.; Woo, J.T.; Sinn, D.H.; Chin, S.O.; Chon, S.; Kim, Y.S. Blood lead is significantly associated with metabolic syndrome in Korean adults: an analysis based on the Korea National Health and Nutrition Examination Survey (KNHANES), 2008. Cardiovasc. Diabetol. 2013, 12, 9. [CrossRef] [PubMed]

15. Wang, X.; Mukherjee, B.; Park, S.K. Associations of cumulative exposure to heavy metal mixtures with obesity and its comorbidities among U.S. adults in NHANES 2003-2014. Environ. Int. 2018, 121 Pt 1, 683-694. [CrossRef]

16. Luzhetskyi, K.P.; Ustinova, O.Y.; Shtina, I.E.; Vekovshinina, S.A.; Ivashova, Y.A.; Tsinker, M.Y. Lipid metabolism changes in population residing in area influenced by storage of ore-processing waste containing lead, cadmium and arsenic. Med. Tr. Prom. Ekol. 2016, 8, 32-37.

17. Kawakami, T.; Nishiyama, K.; Kadota, Y.; Sato, M.; Inoue, M.; Suzuki, S. Cadmium modulates adipocyte functions in metallothionein-null mice. Toxicol. Appl. Pharmacol. 2013, 272, 625-636. [CrossRef]

18. Tyrrell, J.B.; Hafida, S.; Stemmer, P.; Adhami, A.; Leff, T. Lead (Pb) exposure promotes diabetes in obese rodents. J. Trace Elem. Med. Biol. 2017, 39, 221-226. [CrossRef]

19. Vidal, A.C.; Semenova, V.; Darrah, T.; Vengosh, A.; Huang, Z.; King, K.; Nye, M.D.; Fry, R.; Skaar, D.; Maguire, R.; et al. Maternal cadmium, iron and zinc levels, DNA methylation and birth weight. BMC Pharmacol. Toxicol. 2015, 16, 20. [CrossRef]

20. De Luis, D.A.; Izaola, O.; Aller, R. Association of G1359A polymorphism of the cannabinoid receptor gene (CNR1) with macronutrient intakes in obese females. J. Hum. Nutr. Diet. 2016, 29, 118-123. [CrossRef]

21. Manriquez, V.; Aviles, J.; Salazar, L.; Saavedra, N.; Seron, P.; Lanas, F.; Fajardo, C.M.; Hirata, M.H.; Hirata, R.D.C.; Cerda, A. Polymorphisms in genes involved in the leptin-melanocortin pathway are associated with obesity-related cardiometabolic alterations in a southern Chilean population. Mol. Diagn. Ther. 2018, 22, 101-113. [CrossRef] [PubMed]

22. Nowzari, Z.; Masoumi, M.; Nazari-Robati, M.; Saavedra, N.; Seron, P.; Lanas, F.; Fajardo, C.M.; Hirata, M.H.; Hirata, R.D.C.; Cerda, A. Association of polymorphisms of leptin, leptin receptor and apelin receptor genes with susceptibility to coronary artery disease and hypertension. Life Sci. 2018, 207, 166-171. [CrossRef] [PubMed] 
23. Szymańska-Chabowska, A.; Łaczmański, Ł.; Jędrychowska, I.; Chabowski, M.; Gać, P.; Janus, A.; Gosławska, K.; Smyk, B.; Solska, U.; Mazur, G.; et al. The relationship between selected VDR, HFE and ALAD gene polymorphisms and several basic toxicological parameters among persons occupationally exposed to lead. Toxicology 2015, 334, 12-21. [CrossRef] [PubMed]

24. Chen, X.; Dai, Y.; Wang, Z.; Zhu, G.; Ding, X.; Jin, T. The association between serum vitamin D levels and renal tubular dysfunction in a general population exposed to cadmium in China. PLoS ONE 2018, 13, e0195682. [CrossRef]

25. Suárez-Pinilla, P.; Roiz-Santiañez, R.; Ortiz-García de la Foz, V.; Guest, P.C.; Ayesa-Arriola, R.; Córdova-Palomera, A.; Tordesillas-Gutierrez, D.; Crespo-Facorro, B. Brain structural and clinical changes after first episode psychosis: Focus on cannabinoid receptor 1 polymorphisms. Psychiatry Res. 2015, 233, 112-119. [CrossRef]

26. Arikan, T.A.; Kelles, M. Plasma selenium and cadmium levels in patients with chronic otitis media in a Turkish population and their relation to inflammation markers. Biol. Trace Elem. Res. 2019, 189, 55-63. [CrossRef]

27. Rabha, R.; Ghosh, S.; Padhy, P.K. Indoor air pollution in rural north-east India: Elemental compositions, changes in hematological indices, oxidative stress and health risks. Ecotoxicol. Environ. Saf. 2018, 165, 393-403. [CrossRef]

(C) 2020 by the authors. Licensee MDPI, Basel, Switzerland. This article is an open access article distributed under the terms and conditions of the Creative Commons Attribution (CC BY) license (http://creativecommons.org/licenses/by/4.0/). 